\title{
35
}

\section{ObjectMap: Integrating high performance resources into a distributed object-oriented environment}

\author{
Mike Sharrott, Stuart Hungerford and John Lilleyman, \\ Co-operative Research Centre for Advanced Computational Systems (ACSys) \\ CSIRO Division of Information Technology, \\ GPO Box 664, Canberra, ACT 2601, Australia.
}

\begin{abstract}
As modern computing environments mature towards a distributed object-oriented architecture, the ability to effectively integrate a high performance computation resource into the environment becomes more difficult. This is due to the restricted operating system that such resources typically provide with respect to object-oriented programming techniques and distributed access. For similar reasons, the capability of the resource to access modern, persistent object-oriented databases is also limited.

This paper describes the ObjectMap software framework which is currently being developed to address these problems and to more efficiently integrate computation resources into main stream computing. The work focuses on data handling between persistent databases and computation resources across a network.

ObjectMap has adopted the emerging Common Object Request Broker Architecture (CORBA) standard from the Object Management Group (OMG) for its base architecture with the implementation being realised using the Orbix product from Iona Technologies. The paper describes the benefits obtained through using the CORBA and also the limitations that it has introduced.

The research is being performed by the CSIRO Division of Information Technology as a project within the Co-operative Research Centre (CRC) for Advanced Computational Systems (ACSys) in collaboration with the Australian National University's department of Computer Science. It is also being sponsored by Digital Equipment Corporation (Australia) Pty. Ltd.
\end{abstract}

\section{KEYWORDS}

CSIRO, DIT, ObjectMap, OMG, CORBA, Orbix, OODB, ObjectStore 


\section{INTRODUCTION}

Over the past few years, research has been undertaken at the CSIRO's Division of Information Technology (DIT) to allow distributed applications to efficiently access the Division's DECmpp 12000 (MasPar MP-1) massively parallel computer [1, 2, 3]. This research has highlighted the need for a software framework that can transparently handle data originating from a modern persistent object store and make it available for processing on a computation resource. Any such framework should encompass the benefits from an object oriented approach and a distributed architecture.

DIT is developing the software framework, through the ObjectMap CRC project, to be a suitable architecture to address these problems and to provide a test bed on which to experiment with some different data handling, mapping and caching policies.

The project will deliver a demonstration system, shown in Figure 1, which will consist of a client application accessing image data from an Object-Oriented DataBase (OODB) and performing image processing operations on a Massively Parallel Processor (MPP). The client will run on a PC under Windows NT (and possibly Chicago). ObjectStore is being adopted for the OODB which will be hosted by a Sun Sparc workstation. The MPP is a DECmpp 12000 hosted by a Digital Alpha.

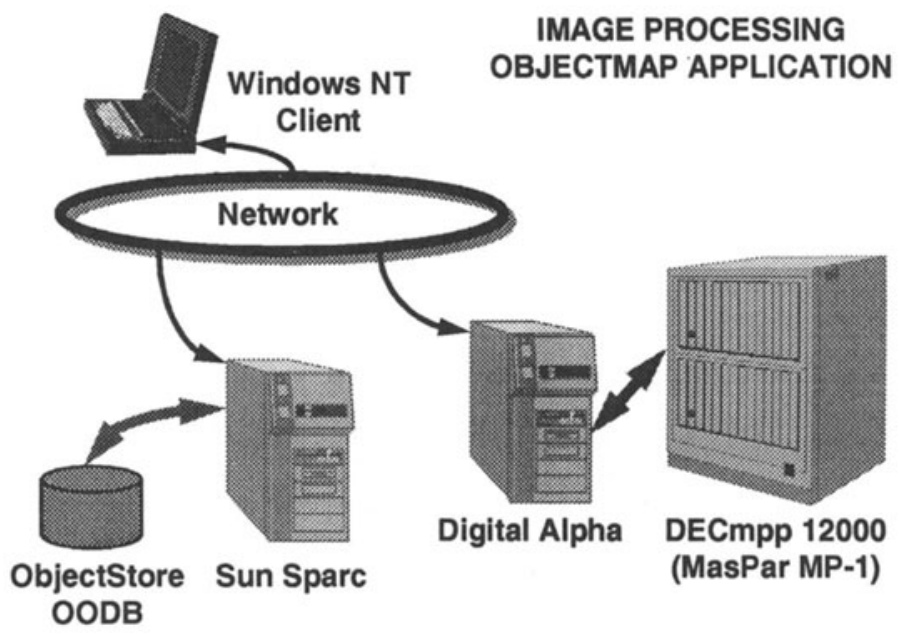

Figure 1 ObjectMap Demonstration System

This paper starts with a discussion of the main requirements that a suitable software framework must meet in order to achieve high efficiency and performance. This is followed by a description of the approach used by ObjectMap and its design components. A summary of the limitations and experiences encountered during the project is also given. 


\section{SOFTWARE FRAMEWORK REQUIREMENTS}

One of the key requirements for a software framework to effectively integrate a computation resource into a distributed object-oriented environment is the need for efficient data handling.

A typical application session involves the client locating the data that it requires (from one of many stores available on the network) then making it available to a computation server where the required operation will be performed. This process possibly involves several data movements and is made worse by the typical requirement of a computation resource to load the data onto its custom processors before the operation may be performed.

These data movements must be kept to a minimum in order to keep the system efficiency, and hence performance, high. The policies adopted for this are store and resource dependent, hence the software framework should provide a solid test-bed on which different policies may be investigated.

The framework should provide access to different kinds of data from a wide variety of stores. These include files from a standard filesystem, $\mathrm{C}++$ or CORBA objects from an Object Oriented DataBase (OODB) and records from a Relational DataBase (RDB). Features should be incorporated to enable the user to browse the data independently of the associated store.

A further requirement of the framework is that it supports multiple users and multiple user access to the computation resource even when the resource itself may not. Either the framework or the resource should have a mechanism for swapping out jobs or segmenting memory to enable multi-user access.

As well as the specific requirements, there are a number of more general features that any such distributed framework should fulfil. The framework should be robust and incorporate exception handling such that it can handle network nodes becoming unavailable during a session. The system should be modular to allow new applications to easily be incorporated. The Graphical User Interface (GUI) of the application client should be able to remain sensitive whilst framework operations are in progress. This allows users, if required, to interrupt the currently active operation or to start setting up the next operation request. These general requirements may be met by adopting a standard core distributed processing package.

\section{THE OBJECTMAP APPROACH}

This section describes the general approach that has been adopted by the ObjectMap framework in order to meet the requirements outlined in Section 2.

\subsection{Object-Oriented (OO)}

A high level abstraction of the requirements for the ObjectMap framework identifies four main components to the system; data servers, data objects, computation servers and application clients. A data server accesses and manages data from a store, making it available as data objects to the other framework components. A computation server performs operations on the data objects using its associated high performance resource. Finally, an application client can browse and select data and operations from the data and computation servers respectively.

By adopting an $\mathrm{OO}$ approach, each of these components map to an object. This enables the complexity of the framework to be hidden through inheritance from core objects. 


\subsection{Distributed Technology and CORBA}

Typically, on a network, the host machines for the data store and for the computation resource are not the same. This means that the ObjectMap framework requires to have distributed technology incorporated in order to allow the components to talk to each other.

Currently the technology of distributed object computing is expanding rapidly due to the release of the Common Object Request Broker Architecture (CORBA) standard [4] from the Object Management Group (OMG). This architecture is designed to allow objects to communicate across heterogeneous platforms whilst still separating the object's interface from its implementation. The CORBA is the communication core of the OMG's larger Object Management Architecture (OMA) [5] which defines a standard set of object services and common facilities that should be provided in a computing environment.

By adopting the CORBA as the underlying communication mechanism, the ObjectMap framework can take advantage of the features that the technology offers. These include a dynamic binding interface which enables client objects to make requests on server objects with no prior compile-time knowledge of the server object's interface or location. This information may be obtained dynamically through repositories on the network. A further feature of the dynamic interface is the ability to make asynchronous function calls between objects.

A disadvantage to the CORBA is that it only deals with CORBA objects which must be defined using the Interface Definition Language (IDL). ObjectMap requires to access other kinds of data from stores, such as $\mathrm{C}++$ objects and file data. Hence the ObjectMap design needs to provide a method for mapping these objects into CORBA data objects whilst still retaining efficient data handling.

There are several implementations of the CORBA available (or soon to be released). These include Orbix (Iona Technologies), ObjectBroker (Digital), Distributed Objects Everywhere (SunSoft) and the System Object Model (IBM). For the first prototype of ObjectMap, it was decided to use Orbix $[6,7,8]$ as it was the most established and robust CORBA implementation available. Orbix also provides a $\mathrm{C}++$ binding (that has been submitted to the OMG as the standard $\mathrm{C}++$ binding [9]) which simplifies application development and maps well to the ObjectMap requirement to access $\mathrm{C}^{++}$object data from persistent stores.

\subsection{Data Objects and Factories}

Typically a data server will access many data components from its store and make them available as data objects. There are several options for managing these data objects ranging from each data object having its own process to all the objects sharing the same process. The optimal approach is application dependent.

The approach used by the ObjectMap framework is to provide a factory object which manages the creation and deletion of the data objects. By this mechanism different options may be tried in order to find the optimal solution for the application.

Many high performance computation resources require that the source data be loaded onto their custom processors before an operation may be performed. Similarly to make the resultant data available at the network level, it must be transferred back from the processors. Typically the resources provide some high bandwidth mechanism for this data movement between the host and the processors. By adopting the factory object to manage the data objects, the ObjectMap framework is able to take advantage of these communication channels. 


\section{OBJECTMAP COMPONENTS}

The ObjectMap architecture, shown in Figure 2, is split into a number of key components.

- ObjectServer

- ObjectFactory

- ComputeServer

- DemoClient

Each of these components are separate CORBA processes and hence may be distributed across a heterogeneous network with CORBA handling the communication. Each component is a single CORBA object with the exception of the ObjectFactory, which consists of multiple CORBA objects: a Factory and a number of DataObjects.

\subsection{ObjectServer}

The Object Server provides the public interface to an associated persistent store of data, which may be a filesystem, OODB or RDB.

By making calls to ObjectFactory objects, the ObjectServer makes data available from the store as CORBA objects called DataObjects (shown in Figure 2 as Data 1 and Data 2). These DataObjects may then be accessed by other CORBA clients. By the same process, the ObjectServer allows new or modified data objects to be incorporated back into the store.

The ObjectServer allows efficient data handling and caching policies to be included between the store and the DataObjects.

\subsection{ObjectFactory}

The ObjectFactory is split into a Factory object and a number of DataObjects (shown in Figure 2 as Data 1 and Data 2). The Factory is responsible for creating and maintaining the DataObjects in response to requests by an ObjectServer. The DataObject is the network representation of a data component from a persistent store. The DataObject communicates with its associated ObjectServer in order to access its data from the store which may involve data copying and caching policies.

\subsection{ComputeServer}

A ComputeServer performs computation on DataObjects in response to a request from a client. The request specifies the required computation parameters including object references to source and destination DataObjects.

Typically the ComputeServer will perform the computations on an associated high performance resource such as a Massively Parallel Processor (MPP), e.g. DECmpp 12000 (MasPar MP-1). This may require the ComputeServer to move or copy data from the DataObjects to the custom processors of the resource before the computation may be performed. This will take advantage of any high performance IO gateways into the resource.

\subsection{DemoClient}

The DemoClient is the application and ObjectMap client and typically provides an eventdriven GUI. The DemoClient binds to ObjectServers in order to retrieve data from a persistent 
store or to create new data which maps to the store. The DemoClient does not see the ObjectFactory but deals directly with references to DataObjects. The DemoClient may also bind to ComputeServers to perform computations on the data. At any stage the DemoClient may be attached to multiple ObjectServers and ComputeServers.

The ObjectMap architecture allows the DemoClient to remain sensitive whilst any ObjectServer or ComputeServer operations are in progress.

\section{OBJECTMAP ARCHITECTURE}

This section provides further details on the design of the individual components of the ObjectMap architecture.

\subsection{ObjectServer}

\subsubsection{Architecture}

The design of an ObjectServer, shown in Figure 3, has adopted a layered architecture for both the object interface and its internal structure.

The object interface is split into two parts:

- Core Functions

- Data-Specific Functions

The core functions must be implemented by all ObjectServers and must provide the base interface necessary to identify and provide general access to the ObjectServer and the data that it serves. The data-specific interface functions provide more efficient access to the data via individual functions for each served data type.

The internal structure of the ObjectServer is layered to adopt maximum portability and reusability across ObjectServers. For example all store specific operations are held within a StoreManager object. This allows the ObjectServer to be mapped from one store to another (e.g. Filesystem to OODB based) simply by rewriting the StoreManager object and not the whole ObjectServer.

\subsubsection{Data Handling}

When a client requests data, the ObjectServer calls a function in the ObjectFactory to create a DataObject of the required type. However, at this point, the data will not be loaded from the store into the DataObject. The ObjectServer will simply maintain a record consisting of a handle to the data in the store and an object reference to its associated DataObject.

When the client (or a different client) binds to the DataObject and calls a member function, the DataObject will recognise that it has not yet been initialised and will bind to the ObjectServer in order to do so. At this point the ObjectServer will access the required data from the store and will transfer it to the DataObject.

Using this handling mechanism, a data caching policy may be inserted between the store and the DataObject, such that the DataObject only contains a working subset of the data that it requires. Using the underlying core functionality, the ObjectMap framework provides a testbed on which to experiment with different data caching and handling policies. 


\subsubsection{Object Pointers}

If the store associated with an ObjectServer is an OODB, then it is likely that the store contains trees of objects where one object may point to several others. This creates a problem when one such object is activated in how to handle the child objects. There are several approaches possible which range from making the requested object and all of its dependants active to making only the requested object active. ObjectMap has adopted the latter approach.

The reason for ObjectMap's "make active only on demand" approach is efficiency and the granularity of the objects proposed to be used in the framework. Because each data component is likely to be large (satellite images or 3D medical data sets) then making the object, and all of its dependant objects active, is likely to use up all the system's resources.

When an object, which has child objects, is accessed from a store, then the associated DataObject must mark its child pointers to signify that they do. not yet have their own DataObject. Hence if a client tries to access a child pointer, the DataObject will first create a new DataObject for the child before processing the request. This is achieved through calls to the Factory back to the ObjectServer.

\subsubsection{Persistence}

The OMG is currently defining a standard for CORBA object persistence through the definition of the standard object services. This will enable a CORBA object to be mapped between being active and being swapped to disk whilst still retaining all state information. This occurs invisibly to the client.

The ObjectServer is providing a similar persistence service for non-CORBA objects. For example, an ObjectServer may handle $\mathrm{C}++$ objects from an OODB or objects stored in a custom binary format in a filesystem.

\subsection{ObjectFactory}

\subsubsection{Architecture}

The ObjectFactory consists of a single Factory object and a number of DataObjects. The Factory is responsible for the creation and deletion of the DataObjects.

When a DataObject is created, it is assigned a unique identifier (UID) which is used for all communications with the ObjectServer in order to uniquely identify the object's associated data in the store. The data handling between a DataObject and its entry in a store is described in Sections 5.1.2 and 5.1.3 above.

\subsubsection{DataObjects and Processes}

In a network environment it may be required to split the DataObjects over several nodes for efficiency purposes. For example, by mapping the DataObject to the host of a computation resource, the resource may be able to take advantage of a high bandwidth path to load the data onto its custom processors. This is described further below in Section 5.3.2. To support this, each DataObject requires to be mapped to its own process so they may be split over the network. The ObjectMap framework provides policies in the Factory object to control the DataObject to process mapping. 


\subsection{ComputeServer}

\subsubsection{Architecture}

The ComputeServer interface is split into two main components:

- Core Functions

- Computation-Specific Functions

The core functions must be implemented by all ComputeServers and will be used by clients whilst browsing several ComputeServers to locate the operations that they require. The core functions identify the ComputeServer and list the operations that it provides. The computationspecific functions provide access to the actual computation operations. The function arguments specify all the required operation parameters including object references to the source and destination DataObjects.

\subsubsection{Data Mapping}

A high performance computation resource typically loads all computation data onto its custom processors before the operation may be performed. Similarly the resultant data is transferred back off the custom processors to the host before it can be accessed at the network level. These data transfers require to be optimised in order to ensure that the performance of the resource still remains high. Most computation resources provide a high bandwidth IO connection between the host and the custom processors. For example, the DECmpp 12000 (MasPar MP-1) [10] provides a RAM based file system (accessible to the network through NFS) which has a connection directly to the parallel processors. By configuring the DataObjects to actually store their data in this filesystem, the full bandwidth connection may be utilised.

For some applications, the system performance may be further improved by configuring the ComputeServer to cache as much computation data as possible on the custom processors pending possible further requests

The ObjectMap framework provides the core functionality such that it may be used as a test-bed for research to be performed into more efficient mappings of DataObjects between the network and the computation resources.

\subsubsection{Parallel Computation Service (PCS)}

High performance computation resources are generally single user machines where one user grabs the whole system for the duration of their work. Other users must queue to access the system.

Research has been carried out at DIT to look at how a system may be made multi-user by providing a job manager. This manager schedules jobs and controls all job swapping as well as providing mechanisms for optimal data handling on the custom processors This research resulted in the Parallel Computation Service (PCS) [1] being developed. A second design of PCS is now underway which is based on the CORBA rather than UNIX socket and pipe connections.

The PCS integrates with the ObjectMap framework seamlessly by configuring each ComputeServer to be a PCS client. Further information on the PCS may be found in [1]. 


\subsection{DemoClient}

\subsubsection{ObjectServer and ComputeServer Browsing}

Being the user interface to an application, the DemoClient requires to be able to browse ObjectServers and ComputeServers in order to locate the data and services that it requires. ObjectMap provides this functionality through a combination of the core interfaces of the servers and the standard CORBA object location and binding services.

\subsubsection{Sensitive User Interface}

Typically the computations being performed by a ComputeServer will not be real time. Hence it is important that the user interface of the DemoClient remains sensitive to user requests whilst operations are in progress. This allows the user to start building the next request or to interrupt the currently active operation. This is achieved in the ObjectMap framework by implementing the function calls asynchronously using the CORBA dynamic binding interface.

In order to notify the DemoClient that a computation has completed, the framework provides two mechanisms of call-back. If the DemoClient is itself a CORBA object, then the ComputeServer can be configured to call a function within the DemoClient on completion. Alternatively the DemoClients may poll a computation status flag within the ComputeServer in order to wait for completion. This polling may be integrated with the event loop of the DemoClient. Further mechanisms for notification will be investigated during the course of the project.

\section{OBJECTMAP LIMITATIONS}

A number of limitations have been identified with the ObjectMap framework. These can be attributed to both the design, the probable applications and to the CORBA. The following sections summarise these findings.

\subsection{Interface Definition Language (IDL)}

The IDL language does not yet provide some of the constructs that would be very useful. In particular, there is no one-to-one mapping between $\mathrm{C}++$ and IDL, hence trying to represent $\mathrm{C}++$ objects as CORBA objects creates several problems. This is not something that the CORBA was designed for as the CORBA assumes that the starting point was an IDL interface.

With respect to $\mathrm{C}^{++}$, the following constructs are missing:

- No constructors or destructors

- No function overloading

- No concept of "by pointer" or "by value"

- No operator functions

- No templates

These limitations may be addressed in future versions of IDL as the technology becomes more established. 


\subsection{Object Granularity}

If an application consists of hundreds of objects of different types, then the overhead costs involved in integrating it with the ObjectMap framework may be quite high. This is made worse if the objects are highly interconnected through pointers and object references.

The ObjectMap framework is biased towards applications consisting of fairly large granularity objects (e.g. satellite images or 3D medical data sets) where typically less than twenty were active at any one time.

One specific application that did not map well to the ObjectMap framework required to access and process many small and highly interconnected $\mathrm{C}++$ objects from an OODB. In this case a specific $\mathrm{C}++$ distributed system was more applicable. For example, the ObjectStore OODB provides an automatic method of persistence for object hierarchies, with the kernel handling all data mappings [11]. A further possible approach is to use a distributed $\mathrm{C}++$ class packages such as NetClasses [12].

\subsection{Data Handling}

Care must be taken when handling pointers in the Orbix implementation of CORBA. For example, if an object member function takes a pointer argument, then Orbix will automatically copy the whole of the "pointed to" data to the address space of the destination object as part of the function call. A similar copy process occurs when a function returns a pointer but with the added limitation that Orbix automatically deletes the function pointer and what it points to after the return has completed. Hence if the function copy is required to be kept then it must be copied prior to the function return.

Obviously at some stage the data requires to be copied across the network between the source and destination object's address spaces, but these movements should be minimised in order to prevent thrashing.

\subsection{Deadlock}

If a CORBA object is processing a request from one client and then it receives another request from a different client, then the second request will be blocked pending completion of the first. Hence if any circular dependencies are present, a deadlock will occur. This may be avoided to a limited extent by making use of threads and time-outs, but this is a general limitation which is present in most distributed or parallel systems.

\section{CONCLUSIONS}

The ObjectMap software framework addresses the main requirements that are necessary for the integration of a high performance computation resource into a distributed object-oriented environment. It also provides a sound test-bed on which different data caching and handling policies may be tested. By taking the object-oriented approach, the ObjectMap framework will hide much of the complexity through inheritance.

The CORBA has provided a sound model on which to develop the framework. In particular the distributed and dynamic binding features are being extensively used. The Orbix implementation has provided a robust development platform and its $\mathrm{C}++$ binding has greatly simplified the development effort. 


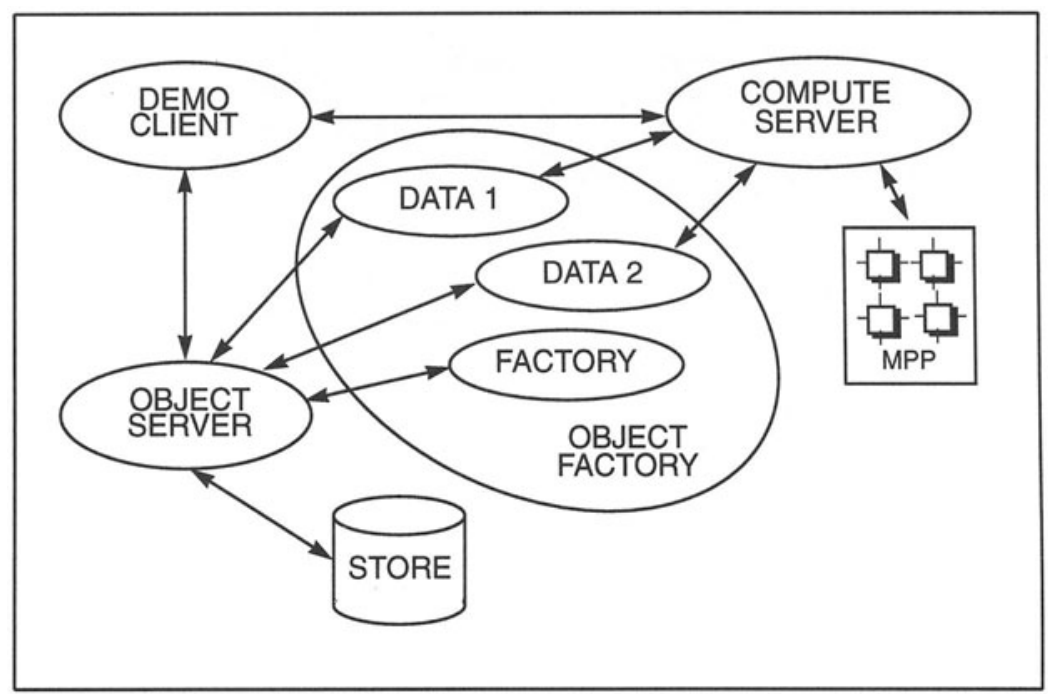

Figure 2 High Level Architecture

Data and Store

Independent

Dependent

Store

Independent

\section{DATA-SPECIFIC}

INTERFACE

Independent

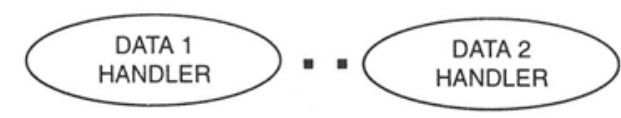

Data

Dependent

Store

Dependent

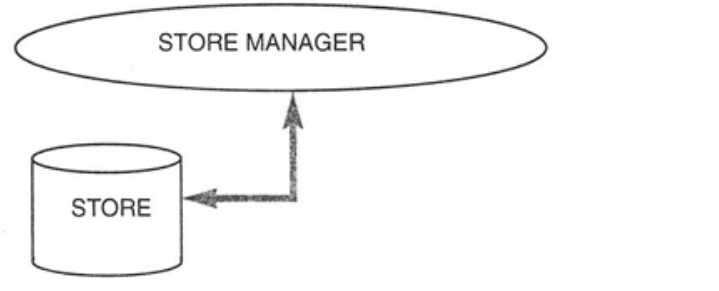

Figure 3 ObjectServer Architecture 


\section{ACKNOWLEDGEMENTS}

The ObjectMap research project is being sponsored by Digital Equipment Corporation (Australia) Pty. Ltd. under the Co-operative Research Centre (CRC) for Advanced Computational Systems (ACSys). This is being performed in collaboration with the Australian National University's department of Computer Science.

\section{REFERENCES}

1. M. R. Sharrott, and J. M. Lilleyman, The Parallel Computation Service (PCS), Proc. DICTA-93, Digital Image Computing: Techniques and Applications, Macquarie University, Australia, December 1993, vol. 1, pp.390-397.

2. D. Keightley, K. Tsui, J.M. Lilleyman, and K. Moore, A software framework for an applications-driven parallel image processing and display system (PIPADS), Proc. SPIE Conference on Recent Advances in Sensors, Radiometric Calibration and Processing of Remotely Sensed Data, vol. 1938, Orlando, Florida, April 1993, pp. 368-379.

3. K. Tsui, P. A. Fletcher and M. A. Hutchins, PISTON - A Scaleable Software Platform for Implementing Parallel Visualisation Algorithms, Proc. Computer Graphics International '94, Melbourne, Australia, June 1994.

4. Object Management Group, The Common Object Request Broker: Architecture and Specification, Revision 1.1, OMG Document Number 91.12.1, December 1992.

5. Object Management Group, Object Management Architecture Guide, Revision 2.0, OMG Document Number 92.11.1, September 1992.

6. Iona Technologies, Orbix - Getting Started, Version 1.2, February 1994

7. Iona Technologies, Orbix - Programmer's Guide, Version 1.2, February 1994.

8. Iona Technologies, Orbix - Advanced Programmer's Guide, Version 1.2, February 1994.

9. Iona Technologies, Orbix - IDL to C++ Mapping, Version 1.2, February 1994.

10. T. Blank, The MasPar MP-1 Architecture, Proc. CompCon ‘90, 1990, pp. 20-24.

11. Object Design Inc., ObjectStore User Guide: Library Interface, Release 3 for UNIX Systems, December 1993.

12. PostModern Computing Technologies Inc., NetClasses - An Object-Oriented Communications Toolkit, Technology Overview. 